Zealand,email:s.hatcher@auckland.ac.nz, Dennis Handrinos Consultant Psychiatrist, University of Melbourne, Victoria, Australia, Kym Jenkins Consultant Psychiatrist, Department of Psychiatry, Alfred Hospital, Melbourne, Victoria, Australia

doi: $10.1192 / p b .32 .4 .151 a$

\section{An elegy to essay writing}

Benning \& Broadhurst (Psychiatric Bulletin, December 2007, 31, 441-442) raise an important issue with regard to the change in the MRCPsych exam format. In addition to the loss of long case, the new exam discards essays and critical appraisal in theory assessment. The loss of essay, in my opinion, deserves significant mourning.

Essays have traditionally been the only mode of testing logical arguing skills. This is an essential skill for any clinician in psychiatry given the intangible nature of certain domains of our clinical work. In the absence of a well-constructed arguing ability, team working and teaching cannot flourish.

Essays tested contemporary contents, unlike multiple choice questions which were obtained from a bank of questions. The creativity and reasoning abilities of a candidate are largely untested in the new format exam. This means we might get many qualified specialists in the future who read the specified syllabus and managed their time well at Clinica Assessment of Skills and Competencies (CASCs, formerly OSCE exams), though they never had a chance to prove that they are up-to-date with the developments in psychiatry or that they could think critically about a controversial issue in the field. This is a great loss as the aforementioned are important and distinguishing skills for any psychiatrist.

I am a candidate who sat the last of old pattern MRCPsych part 2 exams and, like most of my peers, I spent a substantia amount of time researching the British Journal of Psychiatry, Advances in Psychiatric Treatment and Psychiatric Bulletin, as well as other journals, when preparing for my exams. Journal reading habit was cultivated strongly by essay papers in MRCPsych. This is not the case with multiple choice questions. Factual recall is tested equivalently by both multiple choice questions and essays (Palmer \& Devitt, 2007), but higher order cognitive skills including problem-solving cannot be easily tested by a set of questions (Schuwirth et al, 1996). It is, moreover, everyone's secret that the College uses a bank of questions with a high repetition rate for subsequent exams.

One argument against essay writing is standard of assessment, which could vary widely when an essay is evaluated. Standardisation of assessment could be attempted by structured essay evaluation tools. Removing essay writing completely and replacing it with multiple choice questions is a costly trade-off between assessment standards and abilities tested.

Multiple choice questions may be an easy option if one considers online delivery of exam modules in the future, but whether we need to give up on essay papers is a matter of serious debate. Fast food may be easy and appealing, but cannot solve all nutritional requirements!!

\section{Declaration of interest}

L.P. was awarded Laughlin prize for outstanding performance in old format MRCPsych exam, Autumn 2007. He is also involved in writing a multiple choice questions' book for the new format MRCPsych.

PALMER, E. J. \& DEVITT, P. G. (2007) Assessment of higher order cognitive skills in undergraduate education: modified essay or multiple choice questions? Research paper. BMC Medical Education 7,49

SCHUWIRTH, L.W.T., VAN DER VLEUTEN, C. P. M. \& DONKERS, H. H. L. M. (1996) A closer look at cueing effects in multiple-choice questions. Medical Education, 30,44-49.

ena Palaniyappan Academic Clinical Fellow, Division of Psychiatry, Institute of Neuroscience, Newcastle University, LeazesWing, Royal Victoria Infirmary, Newcastle uponTyne NE1 4LP, email: Lena.Palaniyappan@newcastle.ac.uk

doi: $10.1192 / p b .32 .4 .152$

\section{The art of psychiatry}

I read with interest the article by Benning \& Broadhurst (Psychiatric Bulletin, December 2007, 31, 441-442). Holism has become such a cliché in psychiatry. It is sad that at a time when other specialties are embracing the humanities, psychiatry seems to have started to neglect it.

Psychiatry has made a lot of progress over the last few decades. Paging through psychiatric journals filled with imaging studies and genetic breakthroughs showing remarkable discoveries, one can fully appreciate the changes that have been made. In response to these advances in psychiatry Dr. David J. Hellerstein argues that, 'In exploring these new universes, we need not be only technicians and scientists, but also artists!' (Hellerstein, 2007).

The pressures on seeing patients within specified targets and this affair with all things biological has an impact on our patient care. This reductionist psychiatry with quick consultations and quick fixes fits in with the consumer society of 'just add water and stir'. It is unfair to expect a pill to fix complex psychosocial problems.
It is all well and good to have holistic training, but you also need the support and resources to implement the techniques you have learned. In the proposed New Ways of Working we are expected as doctors to only see the most complicated cases. Hopefully, in this new scheme there will be more time allocated to spend with patients and provide them with a more holistic treatment. Teaching will give the foundation to build from, but without the resources to implement holism they will become forgotten poems.

HELLERSTEIN, D. (2007) The Disappearing Patient. Medscape General Medicine (http:// doctor.medscape.com/viewarticle/560699).

Jon van Niekerk Specialist Registrar in Genera Adult Psychiatry, Rawnsley Building, Manchester Royal Infirmary, Oxford Road, Manchester M13 9WL, email:vzvanniekerk@hotmail.com

doi: 10.1192/pb.32.4.152a

\section{Utility of the electroencephalogram}

While the electroencephalogram (EEG) has been available to psychiatrists for over 30 years, its usefulness in psychiatry remains unclear. One study shows that the yield appears low in psychiatry, particularly for epileptic disorder which is fundamentally a clinical diagnosis (Stone \& Moran, 2003). However, this contrasts with Fenton \& Standage's (1993) finding that $92 \%$ of EEGs were useful in a psychiatric series

We compared the requests from psychiatrists for EEGs with the corresponding report in 186 tests (patient group aged 16 years and above, trial over a 28-month period, target population 924 000). This information is held electronically, but we also inspected the original written request in a quarter of cases.

Clear abnormalities suggesting epilepsy or cerebral dysfunction were found in $15 \%$ of the study cohort ( $9 \%$ of $<65$ years old, $39 \%$ of $\geqslant 65$ years old). We defined a test as being useful if it was either clearly abnormal or clearly normal and was likely to add diagnostic weighting in the context of the information on the request form; this usefulness was found in $37 \%$ of tests (32\% in $<65$ years old, $55 \%$ in $\geqslant 65$ years old). The apparent usefulness was reduced if suspected cases of epilepsy were excluded, which happened in 19\% of tests $(16 \%$ in $<65$ years old, $35 \%$ in $\geqslant 65$ years old).

In terms of abnormal positive results for epilepsy, there were no tests supporting unsuspected epilepsy; however, 7 out of 96 in the younger group and 2 out of 26 in the older group did support suspected cases of epilepsy. For cerebral dysfunction, there were 5 out of 45 suspected 